\title{
Estudio del impacto de un cuestionario de autoevaluación online para el tratamiento de errores experimentales
}

\section{Study of the impact of an online self-assessment questionnaire for the treatment of measurement uncertainties}

\author{
María Dolores Calzada Canalejo ${ }^{1}$, Antonio José Cuesta Vázquez ${ }^{2}$, Antonio \\ Manuel Díaz Soriano ${ }^{3}$, Cristina González Gago ${ }^{4}$, José Muñoz Espadero ${ }^{5}$, \\ Encarnación Muñoz Serrano ${ }^{6}$, Antonio Ortiz Mora ${ }^{7}$, Rocío Rincón Liévana ${ }^{8}$, \\ Cristina Yubero Serrano ${ }^{9}$
}

Fecha de recepción: 19/10/2019; Fecha de revisión: 07/11/2019; Fecha de aceptación: 28/11/2019

Cómo citar este artículo:

Calzada, M.D., Cuesta, A.J., Díaz, A.M., González, C., Muñoz, J., Muñoz, E., Ortiz, A., Rincón, R. \& Yubero, C. (2019). Estudio del impacto de un cuestionario de autoevaluación online para el tratamiento de errores experimentales. Revista de Innovación y Buenas Prácticas Docentes, 8(4), 86-97.

Autor de Correspondencia: jmespadero@uco.es

\section{Resumen:}

La correcta comprensión del origen de las incertidumbres experimentales, así como su tratamiento matemático, resulta fundamental en la formación de cualquier científico, pues permite determinar la precisión de los resultados experimentales. Por este motivo, estos conocimientos forman parte de las competencias relacionadas con las asignaturas de Física de los primeros cursos universitarios. Se ha desarrollado e implantado en la plataforma Moodle un cuestionario de autoevaluación online como actividad de autoaprendizaje enfocada al tratamiento de errores en las prácticas de laboratorio de las asignaturas de Física de primer curso en diferentes grados científico-técnicos. Concretamente, fueron puestos a disposición del alumnado de los Grados de Química y Ciencia y Tecnología de los Alimentos. Para valorar el impacto percibido por el alumnado de estos cuestionarios de autoevaluación se realizaron encuestas previas y posteriores a las actividades, cuyos resultados muestran una alta correlación entre el uso de la actividad y la percepción de su utilidad. De igual forma, se han analizado las estadísticas de uso y las calificaciones proporcionadas por la plataforma Moodle, mostrando que, pese a la positiva percepción del impacto del cuestionario en su aprendizaje, es necesario perfilar la actividad para conseguir un mayor grado de uso.

Palabras clave: Autoevaluación, Física, Errores Experimentales, Online.

\footnotetext{
1 Universidad de Córdoba (España), md.calzada@uco.es; CÓDIGO ORCID: 0000-0001-6394-4760

2 Universidad de Córdoba (España), ajcuesta@uco.es; CÓDIGO ORCID: 0000-0002-4153-9470

3 Universidad de Córdoba (España), f62disoa@uco.es; CÓDIGO ORCID: 0000-0003-4486-7303

4 Universidad de Oviedo (España), gonzalezgcristina@uniovi.es; CÓDIGO ORCID: 0000-0002-5040-2628

5 Universidad de Córdoba (España), jmespadero@uco.es; CÓDIGO ORCID: 0000-0001-6383-2910

6 Universidad de Córdoba (España), encarnacion.munoz@uco.es; CÓDIGO ORCID: 0000-0002-21195623

7 Universidad de Córdoba (España), fa2ormoa@uco.es; CÓDIGO ORCID: 0000-0002-5196-7292

8 Universidad de Córdoba (España), rrincon@uco.es; CÓDIGO ORCID: 0000-0003-0298-3365

9 Universidad de Córdoba (España), cyubero@uco.es; CÓDIGO ORCID: 0000-0002-6064-3417
} 


\section{Abstract:}

The correct understanding of the origin of the experimental uncertainties and their mathematical treatment is fundamental in the formation of any scientist, since it allows to determine the precision of the experimental results. For this reason, such knowledge is usually part of the competences related to the Physics subjects of the first university courses. An online self-assessment questionnaire was developed and implemented in the Moodle platform as a self-learning activity focused on the treatment of errors in the laboratory practices of the first year Physics subjects in different scientific-technical degrees. Specifically, it was available to students from Chemistry and Food Science and Technology degrees. To assess the impact perceived by students, surveys were conducted before and after the activities. The analysis of the answers given to those surveys, used as indicators of the perceived impact of the activity, show a high correlation between the use of the activity and the perception of its usefulness. Similarly, usage statistics and the ratings provided by the Moodle platform were analyzed, showing that, despite the positive perception of the impact of the questionnaire on their learning, it is necessary to improve the activity to achieve a greater degree of use.

Key Words: Measurement Uncertainties, Online, Physics, Self-assessment. 


\section{INTRODUCCIÓN}

La comprensión del origen y el tratamiento de las incertidumbres en las medidas experimentales es una materia fundamental en la formación de cualquier científico, ya que además de permitirle establecer una correcta relación entre la teoría y la práctica mediante la verificación experimental de las hipótesis, también le da la posibilidad de determinar el grado de certeza de su medida experimental.

Resulta por tanto indispensable la correcta comprensión de los fenómenos causantes de la aparición de incertidumbres, así como del tratamiento matemático asociado a las mismas. Este es el motivo por el cual, en las asignaturas de Física de los primeros cursos de los grados de orientación científica, se utilizan las prácticas de laboratorio para introducir a los alumnos al estudio del tratamiento de errores experimentales: errores en medidas directas e indirectas, interpolación lineal, media ponderada y regresión lineal, tal y como suele figurar en las guías docentes de dichas asignaturas. Todo esto pone de manifiesto el alto grado de transversalidad y multidisciplinariedad del correcto tratamiento de los errores experimentales.

De la correcta asimilación de dichos conceptos y procedimientos depende en buena medida la calidad del trabajo de un futuro profesional científico, entendido desde una perspectiva amplia para: (1) ser capaz de interpretar correctamente los datos y sus incertidumbres y (2) ser capaz de discernir la calidad de un dato o de los procedimientos utilizados para obtenerlo. Esto está íntimamente relacionado con competencias como la "capacidad de análisis y síntesis" o el "desarrollo del razonamiento crítico", que están presentes en las guías docentes de las asignaturas de física y que aparecen reflejadas entre las competencias básicas de las titulaciones de grado de orientación científica.

Así mismo, como parte del desarrollo de algunas competencias específicas tales como la "capacidad para reconocer y llevar a cabo buenas prácticas en el trabajo científico", la "interpretación de datos procedentes de observaciones y medidas en el laboratorio en términos de su significación y de las teorías que la sustentan" o la "habilidad en el manejo de las magnitudes físicas y sus unidades", se espera del alumnado del grado de ciencias no solo que sea capaz de ejecutar las prácticas de la asignatura con seriedad y rigor científico durante el trabajo en el propio laboratorio, sino también en la fase de análisis e interpretación de los resultados, algo con lo que habitualmente toman contacto por primera vez a través de estas asignaturas.

Por otra parte, investigadores en materia educativa han señalado que los procesos de autoevaluación no solo "poseen fundamentos conceptuales para promover el aprendizaje, así como para mejorar los resultados en las evaluaciones finales" (Carless, Joughin y Mok , 2006), sino que además son una forma de lograr "implicar a los estudiantes en la reflexión sobre qué constituye un buen trabajo" y permitirles "identificar las lagunas y las formas en que pueden cubrirse y dar los pasos necesarios para remediarlo" (Rodríguez, Ibarra y Gómez, 2011), lo que a su vez puede redundar en el desarrollo de las competencias anteriormente citadas.

Es por todo lo anterior que, en el marco del presente trabajo, hemos decidido implantar y evaluar el impacto percibido por el alumnado de un cuestionario de autoevaluación online como herramienta de aprendizaje para el tratamiento de errores experimentales. 
Teniendo en cuenta lo expuesto anteriormente, los objetivos que se han pretendido alcanzar a través del presente proyecto han sido múltiples, dentro del marco del Plan de Innovación y Buenas Prácticas Docentes 2017/2018 de la Universidad de Córdoba. En primer lugar, se ha intentado contribuir al desarrollo de las competencias citadas en el apartado anterior, desarrollando un cuestionario online compuesto por un número suficiente de preguntas. Dicho cuestionario ofrece la posibilidad no solo de poner a prueba sus conocimientos y su capacidad para resolver problemas de tipo numérico, sino también su capacidad para realizar elecciones razonadas en base a dichos conocimientos y su actitud hacia el trabajo científico. También hace hincapié en el rigor y la dimensión ética del trabajo científico, lo cual está estrechamente relacionado con las citadas competencias.

En segundo lugar, a fin de estudiar el impacto percibido por los alumnos del cuestionario de autoevaluación en el proceso de enseñanza-aprendizaje, se han elaborado encuestas anónimas al alumnado. Esto permite incorporar las debidas mejoras y correcciones en años posteriores, abriendo así la puerta a "impulsar las acciones de innovación docente que generen una mejora de los procesos de enseñanza-aprendizaje y de los resultados académicos del alumnado de la UCO", como se pretende en los objetivos del citado Plan de Innovación y Buenas Prácticas Docentes.

Por otra parte, también en consonancia con los objetivos del citado Plan de Innovación y Buenas Prácticas Docentes, la puesta en marcha de un cuestionario de autoevalución contribuye claramente a "potenciar la puesta en práctica de metodologías docentes centradas en la actividad del alumnado", permitiendo al alumno realizar una evaluación autónoma de su grado de conocimiento de la materia. Así mismo, el hecho de que se encuentre disponible online permite "apoyar la incorporación de las tecnologías de la información y comunicación, aplicadas a la enseñanza".

Finalmente, la propia ejecución del proyecto requiere necesariamente del desarrollo de un cuestionario online que, aprovechando las posibilidades de la plataforma Moodle que la Universidad de Córdoba pone a disposición del profesorado, permitirá la exportación del mismo para su reutilización en cursos posteriores o incluso otras plataformas, hecho que contribuye a "promover la producción de material digital y la utilización de los recursos disponibles para ello en la universidad".

\section{DESARROLLO DE LA EXPERIENCIA DE INNOVACIÓN}

En este apartado se describe la experiencia de innovación que se presenta. De igual forma que en el caso anterior, el primer párrafo no lleva sangría.

El segundo párrafo y siguientes presentan una sangría de 1.25. El apartado se puede estructurar en subapartados, que observarán estas mismas reglas.

\subsection{Materiales y Métodos}

El Cuestionario de Autoevaluación se creó utilizando la plataforma Moodle de la Universidad de Córdoba (Moodle 3.1), insertado como una Actividad de tipo Cuestionario generado al azar mediante a partir de un Banco de Preguntas previamente elaborado por el profesorado y cargado en la plataforma. Los 
cuestionarios fueron diseñados para contener una cantidad, tipo de preguntas y ponderación de las mismas similares a los exámenes de tratamiento de datos experimentales de las correspondientes asignaturas. Así mismo, las preguntas fueron diseñadas para que su nivel de dificultad fuese similar al de los citados exámenes.

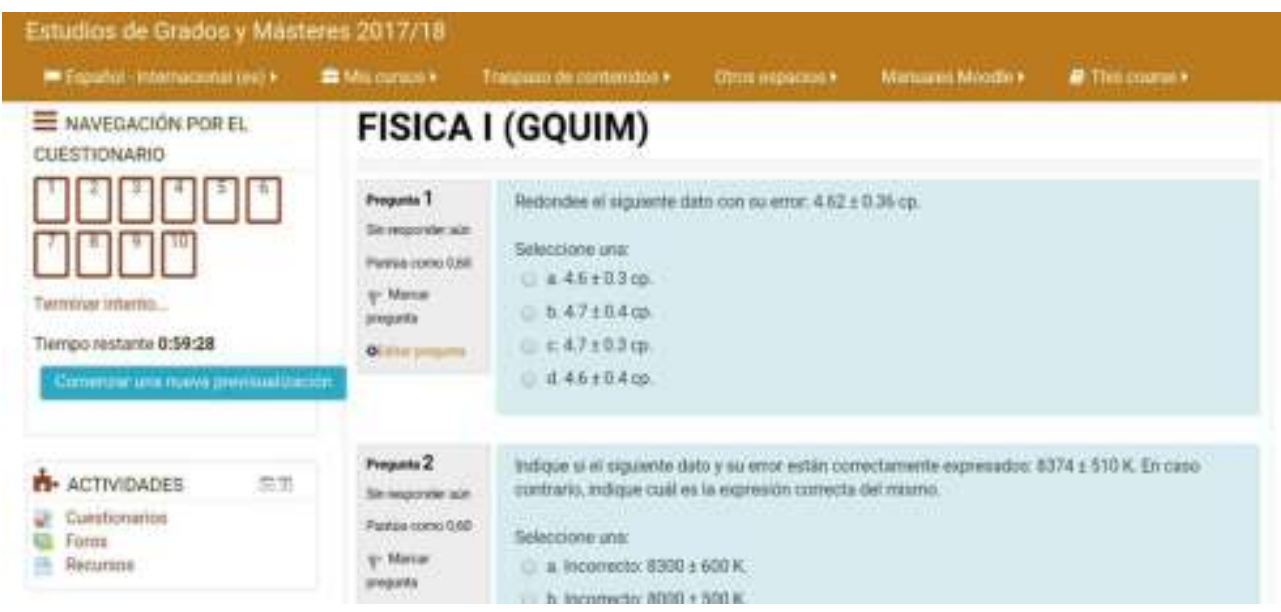

Figura 1.

Captura de pantalla del cuestionario de autoevaluación online en la plataforma Moodle de la Universidad de Córdoba.

\section{Cuestionario Final de Prácticas de Laboratorio de Física}

Valore cada una de las siguientes afirmaciones marcando de 1 a 4 según su grado de acuerdo con ellas, de acuerdo con su experiencia personal, significando (1) totalmente en desacuerdo, (2) en desacuerdo, (3) de acuerdo y (4) totalmente de acuerdo.

\begin{tabular}{|c|c|c|c|c|c|}
\hline & Afirmación & 1 & 2 & 3 & 4 \\
\hline 1 & $\begin{array}{l}\text { Las prácticas de laboratorio de física me han permitido asimilar más fácilmente algunos de los } \\
\text { conceptos explicados en teoría. }\end{array}$ & & & & \\
\hline 2 & Conozco con precisión la diferencia entre los conceptos de dato, error absoluto y error relativo. & & & & \\
\hline 3 & Conozco las causas que provocan la aparición de incertidumbres en las medidas experimentales. & & & & \\
\hline 4 & Conozco al menos un criterio para redondear un dato experimental. & & & & \\
\hline 5 & Conozco al menos un criterio para redondear un error experimental. & & & & \\
\hline 6 & Conozco la diferencia entre una medida directa y una medida indirecta. & & & & \\
\hline 7 & $\begin{array}{l}\text { Conozco la diferencia entre una media aritmética y una media ponderada y comprendo cuándo es } \\
\text { necesario utilizar cada una de ellas. }\end{array}$ & & & & \\
\hline 8 & $\begin{array}{l}\text { Comprendo la necesidad de manejar correctamente los datos experimentales obtenidos durante la } \\
\text { realización de una experiencia de laboratorio. }\end{array}$ & & & & \\
\hline 9 & $\begin{array}{l}\text { Comprendo las implicaciones éticas que se derivan del correcto manejo de los datos del } \\
\text { laboratorio. }\end{array}$ & & & & \\
\hline 10 & $\begin{array}{l}\text { Comprendo la importancia de manejar correctamente unas fuentes de información adecuadas y } \\
\text { contrastar los datos. }\end{array}$ & & & & \\
\hline 11 & $\begin{array}{l}\text { He utilizado el cuestionario de autoevaluación de prácticas de laboratorio de física disponible en } \\
\text { Moodle para la elaboración de las memorias de prácticas. }\end{array}$ & & & & \\
\hline 12 & $\begin{array}{l}\text { El cuestionario de autoevaluación disponible en Moodle me ha resultado útil para la elaboración } \\
\text { de las memorias de las prácticas de laboratorio. }\end{array}$ & & & & \\
\hline 13 & $\begin{array}{l}\text { He utilizado el cuestionario de autoevaluación de prácticas de laboratorio de física disponible en } \\
\text { Moodle para la preparación del examen de prácticas. }\end{array}$ & & & & \\
\hline 14 & $\begin{array}{l}\text { El cuestionario de autoevaluación disponible en Moodle me ha resultado útil para la preparación } \\
\text { del examen de las prácticas de laboratorio. }\end{array}$ & & & & \\
\hline
\end{tabular}

OBSERVACIONES (Indique aquellas sugerencias que, a su juicio, servirían para mejorar la experiencia del cuestionario de autoevaluación de prácticas de laboratorio en particular y, de manera general, el desarrollo de las prácticas de laboratorio de la asignatura de Física):

Figura 2.

Captura de la encuesta anónima postest para la evaluación del impacto percibido por el alumnado del cuestionario de autoevaluación online. 
Al objeto de determinar el impacto percibido por el alumnado en el aprendizaje, se realizaron encuestas anónimas con preguntas sobre la percepción de la asimilación de la materia en cuestión antes (pretest) de los seminarios que cubren dicha materia y de las sesiones de laboratorio, así como después (postest) de los mismos. Dichas encuestas se compusieron de preguntas en escala Likert $1-4$ relacionadas con el grado de percepción de la asimilación de la materia, así como del grado de utilización y la utilidad del cuestionario de autoevaluación online. La Figura 2 muestra las encuestas postest realizadas a los alumnos. Los ítems 11 al 14, ambos inclusive, que se corresponden con la valoración del cuestionario de autoevaluación, no formaron parte de las encuestas iniciales, que solo contuvieron los ítems del 1 al 10, ambos inclusive. Los resultados de dichas encuestas, muy especialmente de los mencionados ítems 11 al 14, fueron utilizados como indicadores del impacto percibido por los alumnos del cuestionario de autoevaluación.

El alumnado objeto de estudio fue aquel de las asignaturas Física I y Física de los grados de Química y de Ciencia y Tecnología de los Alimentos (CyTA), respectivamente, de la Universidad de Córdoba que, durante el curso académico 2017/18, debieron realizar las prácticas de laboratorio de las asignaturas anteriormente mencionadas. En el desarrollo de esta actividad participaron inicialmente 62 alumnos del primer curso del Grado de Química de la Facultad de Ciencias de la Universidad de Córdoba, así como 48 alumnos del primer curso del Grado de Ciencia y Tecnología de los Alimentos de la Facultad de Veterinaria de la Universidad de Córdoba. El nivel educativo de ambos grupos de alumnos es similar ya que, salvo excepciones, provienen en su mayoría de Bachillerato de Ciencias.

\subsection{Desarrollo de la Experiencia}

A fin de alcanzar los objetivos propuestos en el apartado anterior, el profesorado encargado de ejecutar el presente proyecto de innovación docente desarrolló las siguientes actividades. Dado que las asignaturas involucradas se impartieron tanto en el primer como en el segundo cuatrimestre del curso 2017/2018, dichas actividades se dividieron en Actividades Previas, aquellas comunes para todas las asignaturas y anteriores a la implantación del cuestionario online en las que pudo participar todo el profesorado adscrito al proyecto; de Desarrollo, concebidas para ser llevadas a cabo principalmente por el profesorado de las asignaturas, integrándolas en el desarrollo normal de las mismas; y de Finalización, orientadas fundamentalmente al análisis de los datos recabados y la evaluación conjunta de los resultados del proyecto.

Actividades Previas:

- Junio - Agosto 2017: Coordinación del equipo de trabajo para la elaboración de las preguntas que compondrán el cuestionario de autoevaluación online y las encuestas anónimas.

- Semana Anterior al Inicio de la impartición de la asignatura: Incorporación de las cuestiones de autoevaluación en la plataforma Moodle de la asignaturas en las que se pretende desarrollar el presente proyecto (Figura 1).

Actividades de Desarrollo:

- Semanas $1^{\underline{a}}$ y $2^{\underline{a}}$ : Realización de una encuesta anónima de exploración previa sobre los conocimientos y actitudes del alumnado hacia el trabajo 
científico de laboratorio (Pretest). Esta encuesta se realizará antes de la impartición de los seminarios específicos sobre cálculo de errores y tratamiento de datos programados en la asignatura.

- Semanas $3^{\underline{a}}$ a $12^{\underline{a}}$ : Puesta a disposición de los alumnos del cuestionario de autoevaluación online, coincidiendo con el periodo de ejecución de las prácticas de laboratorio. Durante este periodo los participantes en el proyecto se coordinarán para supervisar y resolver las posibles incidencias que puedan surgir relacionadas con el uso del cuestionario de autoevaluación y aplicar las correcciones pertinentes.

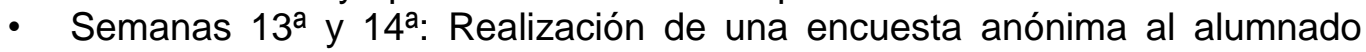
para conocer su percepción del impacto del cuestionario de autoevaluación en el proceso de enseñanza-aprendizaje (Postest).

- Semana 15ª: Evaluación final de las prácticas de la asignatura.

Actividades de Finalización:

- Junio 2018 (Semana $1^{\underline{a}}$ y $2^{a}$ ): Evaluación de los resultados y las encuestas anónimas del alumnado.

- Junio 2018 (Semanas 3a a 4aํ): Redacción y entrega de la memoria final del Proyecto de Innovación Docente.

\section{RESULTADOS}

En primer lugar, se ha estudiado la evolución en la percepción del alumnado del proceso de aprendizaje a través de sus respuestas en encuestas inicial y final, así como de la valoración que realizan del cuestionario de autoevaluación como herramienta para facilitar el estudio y la preparación de la asignatura. De igual forma se ha tratado de extraer información indicativa del impacto percibido de la implantación del cuestionario de autoevaluación online a partir de los resultados y estadísticas de utilización de Moodle.

\subsection{Encuestas anónimas inicial (Pretest) y final (Postest)}

Las Figuras 3 y 4 muestran la evolución de la media de valoración de las afirmaciones 1 a 10 de los cuestionarios pretest y postest para el alumnado del grado de Química y CyTA, respectivamente. A la luz de estos resultados podemos ver que tras la realización de las prácticas de laboratorio y entrega de la memoria de prácticas y del estudio previo al examen usando entre otras herramientas el cuestionario implantado en Moodle, la valoración del alumnado en cuestiones relacionadas con el conocimiento de la terminología o el manejo de los datos experimentales ha aumentado considerablemente en ambas poblaciones objeto de estudio. Esto puede ser concluido ya que el grado de acuerdo aumenta de manera significativa, llegando a valores cercanos a 4, equivalente a totalmente de acuerdo, en casi todas las afirmaciones.

Por otro lado, y con el objeto de evaluar la utilidad del cuestionario de autoevaluación online percibida por los alumnos, se estudiaron de manera independiente las afirmaciones 11, 12, 13 y 14 en el cuestionario postest. Las cuestiones 11 y 13 hacen referencia al uso del cuestionario Moodle para la elaboración de la memoria y el estudio de la asignatura, respectivamente; mientras que la 12 y 14 se centran en estudiar la utilidad percibida en dicha actividad para la elaboración de la memoria de prácticas y para preparar el examen de prácticas, respectivamente. En la Figura 5 se ilustra la correlación existente entre la utilidad del cuestionario implantado en Moodle percibida por el alumnado frente al grado de uso del mismo, tanto para la elaboración de la memoria (arriba) como del estudio de la asignatura (abajo). Tal y como se observa, existe una correlación clara entre el uso del cuestionario Moodle y 
su utilidad, ya que aquellos alumnos/as que afirman haber utilizado más el cuestionario lo encontraron más útil tanto para la elaboración de las memorias como para la preparación del examen práctico de la asignatura.

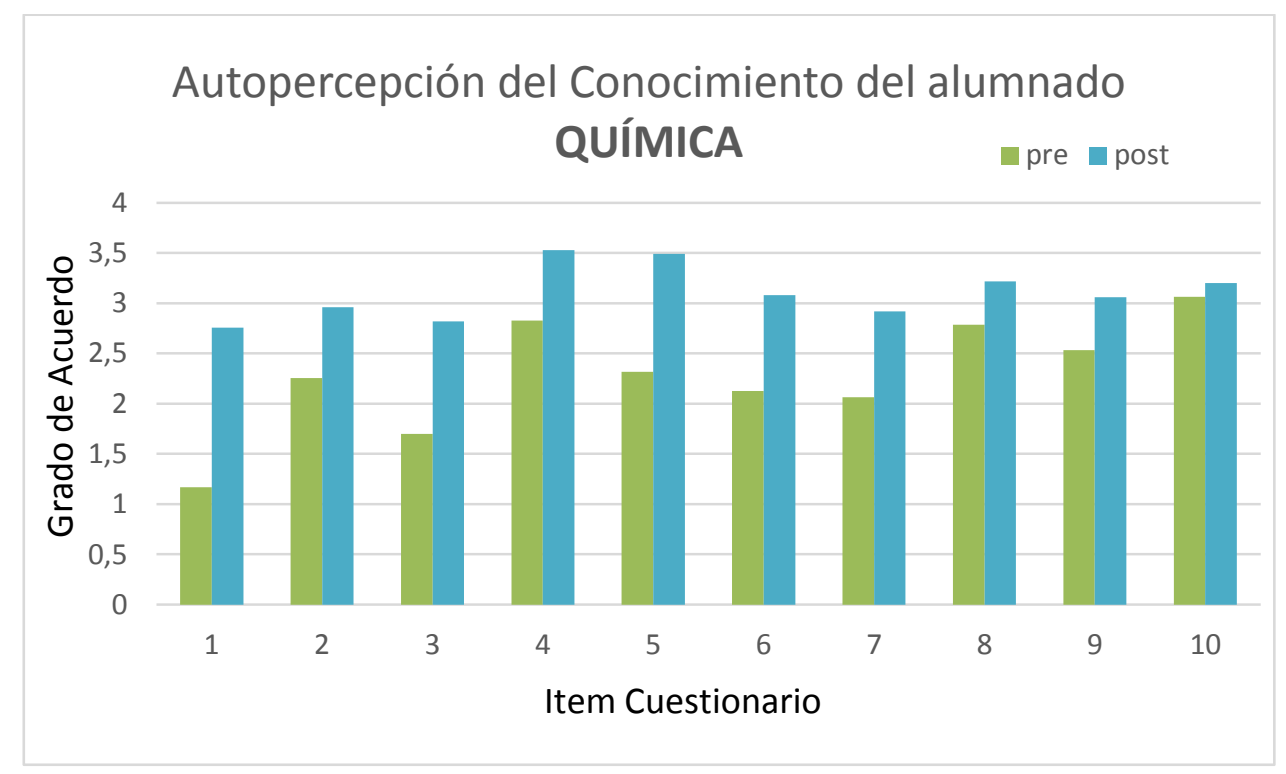

Figura 3.

Comparación de los ítems 1 al 10 de las encuestas previas y posteriores que reflejan la percepción del alumnado de Química de los conocimientos referidos al cálculo de errores y el trabajo científico.

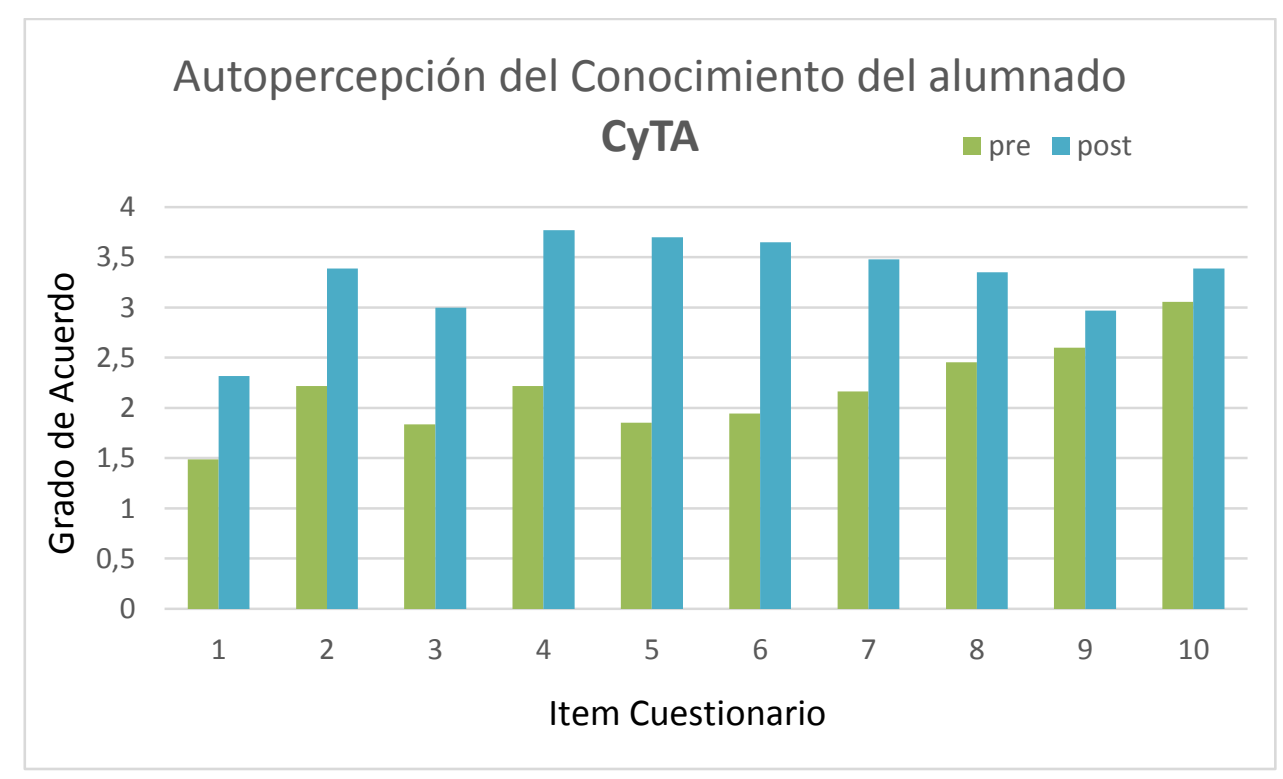

Figura 4.

Comparación de los ítems 1 al 10 de las encuestas previas y posteriores que reflejan la percepción del alumnado de CyTA de los conocimientos referidos al cálculo de errores y el trabajo científico. 

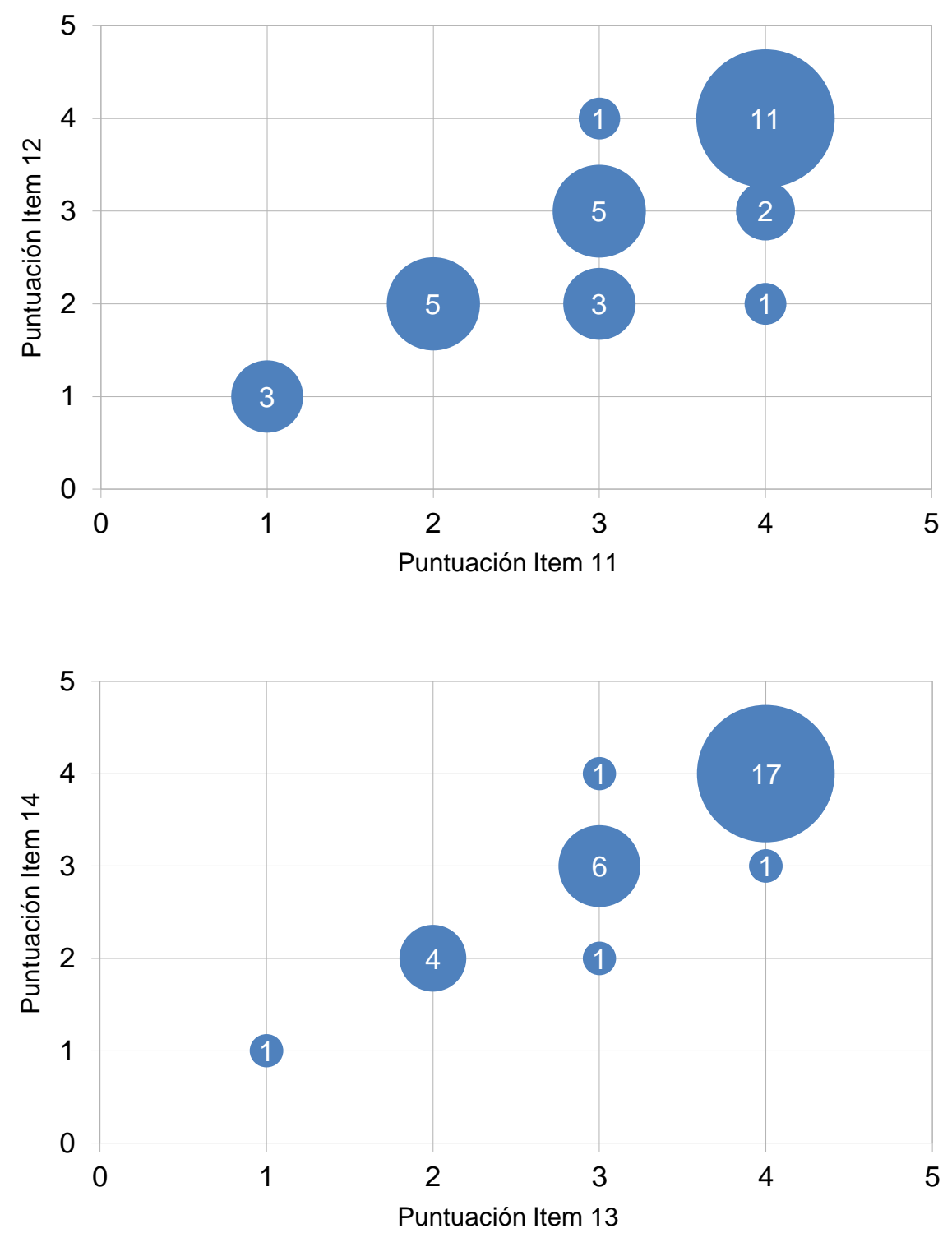

Figura 5.

Diagramas de correlación de la utilidad percibida del test en la preparación de los elementos de evaluación: memorias de prácticas de laboratorio (arriba) y exámen (abajo).

\subsection{Grado de uso del cuestionario de autoevaluación integrado en la plataforma Moodle}

Tal y como se ha explicado, el cuestionario de autoevaluación se implantó en la plataforma Moodle con el fin de facilitar el estudio del alumnado de la asignatura. Si bien los cuestionarios indicaban una nota numérica al alumnado, dicha nota no se utilizó para la evaluación. Además de la retroalimentación en forma de calificación numérica, los cuestionarios indicaban tras su resolución completa qué preguntas eran correctas y las soluciones de aquéllas que hubiesen sido incorrectas, proporcionando así información adicional de cara al estudio de la asignatura.

Tras comenzar un cuestionario el alumno/a puede finalizarlo o dejarlo inacabado. Es importante señalar que el $85.5 \%$ del alumnado en el caso del grado de Química y el $70.8 \%$ en el caso del grado de CyTA intentó realizar los cuestionarios integrados en Moodle en algún momento. Este resultado indica el elevado interés del 
alumnado por practicar los contenidos explicados en el aula de una manera independiente gracias a la posibilidad de obtener las soluciones del ejercicio con la facilidad que las nuevas tecnologías ponen a su alcance. Sin embargo, es significativo que este alto porcentaje de intentos se vea reducido cuando se consideran los alumnos que llegan a terminar el cuestionario. Dicho porcentaje se reduce notablemente en el caso del alumnado de CyTA, quedándose sólo un $50.0 \%$, frente a un $83.9 \%$ en el caso del grado de Química. Es importante destacar que hay un núcleo importante de alumnado que intenta el cuestionario más de una vez, pero un número significativo de alumnos/as que intentan el cuestionario tan sólo una vez y lo dejan inacabado. De este modo, habría que entender por qué estos alumnos/as abandonan los intentos del mismo. Con el objeto de ver la distribución de números de intentos finalizados por alumno/a se ha representado en la Figura 6 esta distribución para el conjunto del alumnado de los grados de CyTA y Química. A la luz de los resultados se observa que si bien hay un alto número de alumnos/as que utilizan el cuestionario una sola vez, la mitad (54\%) de los alumnos/as que finalizan el cuestionario lo han hecho en tres ocasiones o menos mientras que la otra mitad (46\%) realizan cuatro intentos 0 más del mismo.

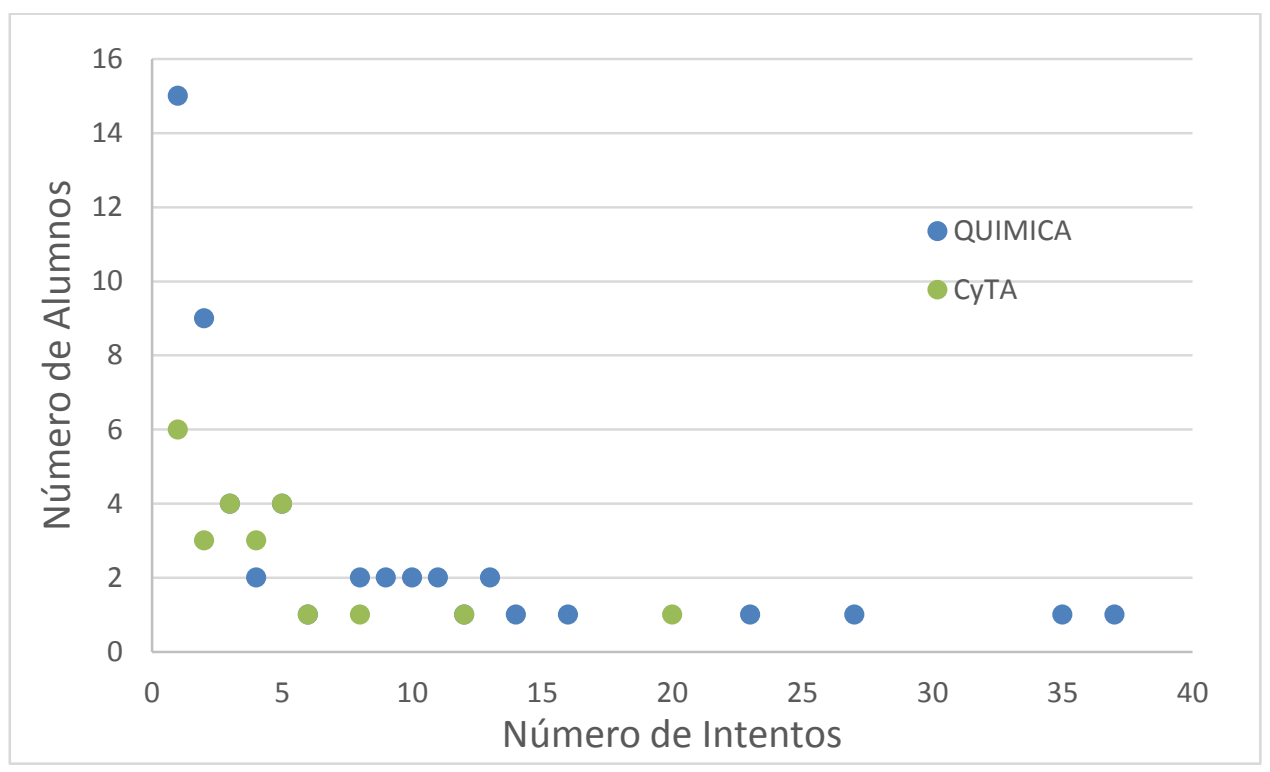

Figura 6.

Relación entre el número de intentos realizados y el número de alumnos/as que los realizan.

Como se ha mencionado, el cuestionario de autoevaluación integrado en Moodle indicaba la nota obtenida en la misma marcando la solución de las respuestas incorrectas. De la calificación de cada uno de los intentos finalizados en la plataforma se puede estudiar la distribución de frecuencias de dichas calificaciones, que se muestra en la Figura 7. Dichos datos indican que de los 371 intentos finalizados entre las dos poblaciones objeto de estudio (alumnado de los grados de Química y de CyTA), 127 de ellos obtuvieron una calificación igual o inferior a 3.99 puntos, frente a los 244 intentos en los que se obtiene una calificación superior o igual a 4. Este resultado es indicativo de que existe un alto número de intentos en los que se obtiene una calificación de aprobado o cercano al aprobado. Es importante notar en este sentido que las mejores calificaciones se alcanzan tras varios intentos, lo que indica 
que el cuestionario desarrollado favorece la adquisición y afianzamiento de los conocimientos relacionados con él.

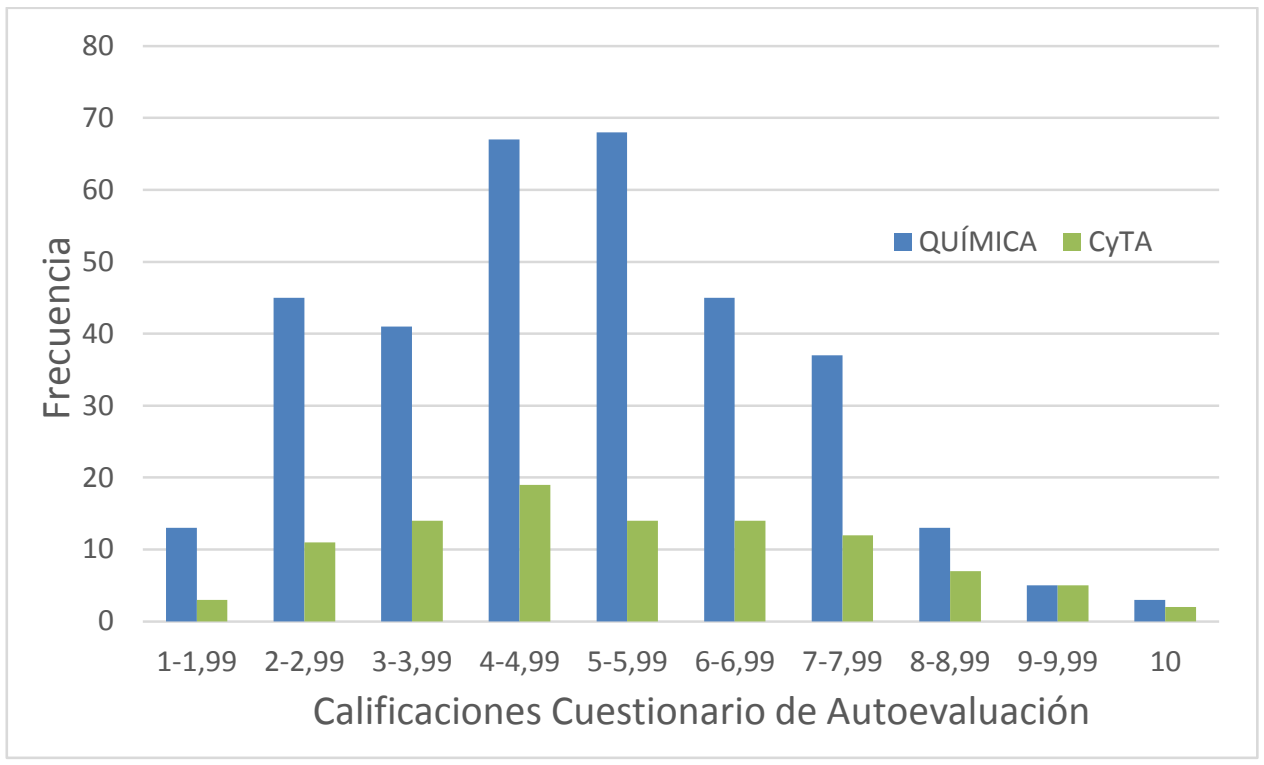

Figura 7.

Calificaciones de los intentos finalizados del cuestionario de autoevaluación en la plataforma Moodle.

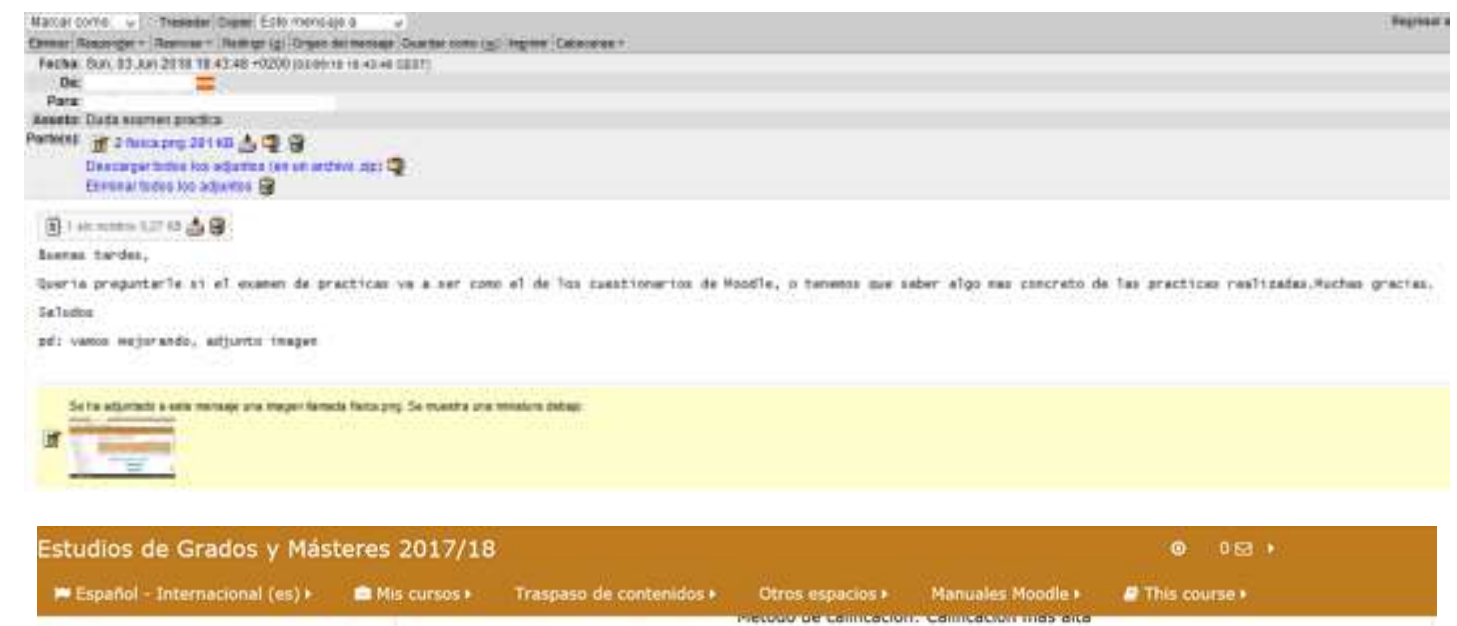

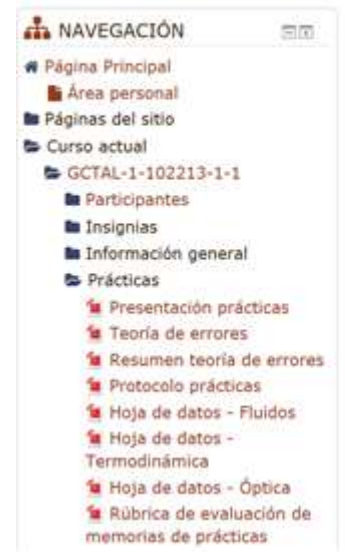

\section{Resumen de sus intentos previos}

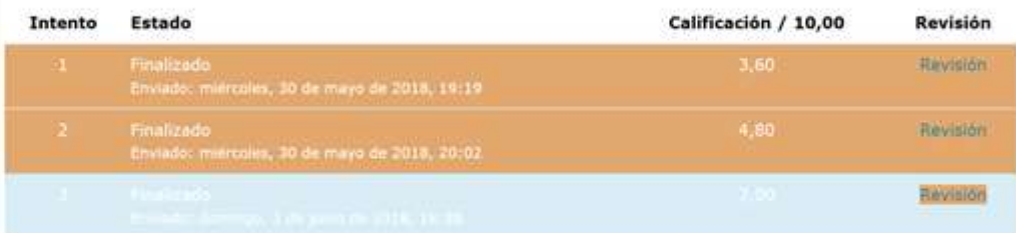

Calificación más alta: 7,00 / 10,00.

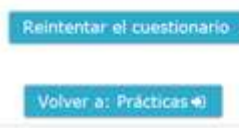

Figura 8.

Retroalimentación del uso del cuestionario Moodle por parte de un alumno/a. 
Precisamente, es la posibilidad de realizar el cuestionario en varias ocasiones lo que hace especialmente atractivo y motivador su uso por parte del alumnado. Este hecho ha sido manifestado por los propios alumnos/as al profesor responsable de las prácticas de la asignatura, que además ha podido constatarlo, verificando que la calificación obtenida se incrementa a medida que aumentaban el número de intentos del mismo. En este sentido, la Figura 8 muestra un correo electrónico enviado por un alumno/a al profesor/a responsable de las prácticas en el que el propio alumno adjuntaba una imagen mostrando el aumento de puntuación tras la realización de varios intentos del cuestionario de autoevaluación de Moodle, indicando en el cuerpo del correo electrónico "pd: vamos mejorando, adjunto imagen", en referencia a su progreso en la interiorización de los contenidos de la asignatura.

\section{CONCLUSIONES}

Del análisis de los resultados arrojados por las encuestas anónimas y las estadísticas de uso del cuestionario de autoevaluación realizado en el apartado anterior se pueden extraer las siguientes conclusiones. En primer lugar, las encuestas realizadas indican una mejora significativa de la autopercepción del aprendizaje en el alumnado, que se debe en parte a la utilización del cuestionario de autoevaluación online, como muestra la elevada correlación entre su grado de uso y la percepción de su utilidad en la elaboración de las memorias de prácticas, así como en exámenes.

Por otra parte, pese al elevado grado de aceptación de la actividad en el alumnado, los resultados muestran no obstante que sería recomendable buscar mecanismos que permitan incrementar su tasa de uso, así como evitar los abandonos de la actividad. Por último, aunque las calificaciones obtenidas por los alumnos en el cuestionario de autoevaluación muestran resultados modestos, estos mejoran significativamente con el grado de uso de la actividad.

\section{AGRADECIMIENTOS}

Este trabajo ha sido financiado a través del proyecto 2017-1-2011 del Plan de Innovación y Buenas Prácticas Docentes 2017/2018 del Vicerrectorado de Estudios de Postgrado y Formación Continua de la Universidad de Córdoba.

\section{REFERENCIAS}

Carless, D., Joughin, G. y Mok, M. (2007) Learning oriented assessment: principles and practice. Assesment and Evaluation in Higher Education, 31 (4), 395-398. https://doi.org/10.1080/02602930600679043

Rodríguez, F. Ibarra, M.S. y Gómez M.A. (2011) e-Autoevaluación en la Universidad: un reto para profesores y estudiantes. Revista de Educación, 356, 401-430. DOI: DOI: 10.4438/1988-592X-RE-2011-356-045 